Dias, L.C., J.N. Clímaco, Exploring the consequences of imprecise information in choice problems using ELECTRE, in: Bouyssou, D., E. Jacquet-Lagrèze, P. Perny, R. Slowinski, D. Vanderpooten e Ph. Vincke (eds), Aiding Decisions with Multiple Criteria Essays in Honor of Bernard Roy, Kluwer, 2002, pp. 175-193.

\title{
EXPLORING THE CONSEQUENCES OF IMPRECISE INFORMATION IN CHOICE PROBLEMS USING ELECTRE
}

\section{Luis C. Dias and João N. Clímaco}

INESC and Faculty of Economics, University of Coimbra, Av. Dias da Silva 165, 3004-512, Coimbra, Portugal.

\begin{abstract}
ELECTRE I, IS and its variants are well-known methods to help Decision Makers (DMs) choose one action (alternative) from a discrete set. Here, we consider the case where the DMs have difficulties in fixing precise values for all the parameters required by ELECTRE, such as the importance of the criteria, the veto thresholds or the performances of the actions. We indicate how to obtain robust conclusions when the DMs specify a set of multiple acceptable combinations of values for the parameters. We argue that robust conclusions should be studied at the outranking relation level, and then suggest some approaches to enrich such conclusions (introducing a tolerance) and to exploit them.
\end{abstract}

\section{INTRODUCTION}

We owe Bernard Roy and his colleagues for the ELECTRE methodology (see Roy, 1991; Roy and Bouyssou, 1993) and the concept of robustness analysis in multicriteria decision aid (Roy, 1998; Roy and Bouyssou, 1993). This paper addresses robustness analysis in the context of ELECTRE methods (ELECTRE I and its variants) for choosing one action from a discrete set considering multiple evaluation criteria.

Using ELECTRE requires setting the value of its input parameters (we use this word in a broad sense), such as the performance of the actions (alternatives), the importance and veto power of the criteria, etc. Providing precise figures for all the parameters is often hard for Decision Makers (DMs): some data may be missing, uncertain, contradictory or imprecise, some modeling options are subject to arbitrariness, and some parameters reflect the values of the DMs, which they may find difficult 
to express and that may change over time (see also Roy, 1989; French, 1995).

To deal with these difficulties, we consider a setting where multiple combination of values for the parameters are accepted, instead of a precise one. This type of information is often named "imprecise" (e.g. Miettinen and Salminen, 1999), "partial" (e.g. Charnetski and Soland, 1978) or "poor" (e.g. Bana e Costa and Vincke, 1995). The multiple combinations of parameter values can be either enumerated or defined by mathematical constraints (provided by the DMs or inferred from holistic comparisons as in Mousseau, 1993).

Robustness analysis concerns the study of the results that are valid for the multiple combinations of input values. We deem that this type of analysis is useful from the very beginning of the decision process. Firstly, it produces information (which conclusions are robust? which results are more affected by the imprecision?) that may guide the DMs in revising the information they provide, progressively narrowing the range of acceptable values for the parameters. Secondly, it allows the DMs to avoid the questions they consider difficult, or at least to postpone these questions until they feel more familiar with the problem and more confident about the answers. In group decisions, accepting multiple combinations of parameter values may foster cooperation among the DMs, as they may accept multiple opinions and may reach consensus on the conclusions that are robust (see Dias and Clímaco, 2000b, in the context of sorting problems). This type of analysis may also be used in the context of an interactive aggregation/ disaggregation approach to decision aid (see Dias et al., 2000, in the context of sorting problems).

This paper discusses how to choose the most preferred action considering imprecise information, within the general context of ELECTRE methods. Previous research concerning choice problems has focused on the multiattribute value function model (for a recent review see Dias and Clímaco, 2000a), with few exceptions. For instance, Roy and Bouyssou (1993) present a real-world study using ELECTRE IS where a robustness analysis was conducted considering a discrete set of combinations of input values; Miettinen and Salminen (1999) present an approach to find the criteria weights (if any exist) that make each certain action the best in terms of the min procedure (Pirlot, 1995).

In the next section we overview ELECTRE's way of constructing and exploiting an outranking relation. In Section 3 we discuss our methodology of finding robust conclusions concerning the outranking relation using optimization. The optimization problems range from very simple ones (ELECTRE I) to the more complicated ones that may appear if credibility indices are to be computed. If few robust conclusions are 
found, then we suggest in Section 4 how to obtain richer (though more fragile) results by introducing a tolerance. Section 5 addresses the exploitation of robust conclusions. We provide an example in Section 6 and we end with a concluding section.

\section{CHOOSING WITH ELECTRE: BRIEF REMINDER}

Consider a set of $m$ actions $A=\left\{a_{1}, \ldots, a_{m}\right\}$ described by their performances at $n$ criteria. Let $g_{j}\left(a_{i}\right)$ denote the performance of $a_{i}$ at the $j$-th criterion. The first phase of ELECTRE consists in building an outranking relation defined over $A$. For each ordered pair of actions $\left(a_{x}, a_{y}\right)$, the method finds whether $a_{x}$ outranks $a_{y}$ (i.e. $a_{x}$ is at least as good as $a_{y}$ ) or not. We will use the following notation for the parameters:

- $\Delta_{j}$ represents the advantage of $a_{x}$ over $a_{y}$ at the $j$-th criterion: $\Delta_{j}=\left\{\begin{array}{l}g_{j}\left(a_{x}\right)-g_{j}\left(a_{y}\right), \text { if the } j^{\text {th }} \text { criterion is to be maximized; } \\ g_{j}\left(a_{y}\right)-g_{j}\left(a_{x}\right), \text { if the } j^{\text {th }} \text { criterion is to be minimized; }\end{array}\right.$

- $k_{j} \geq 0$ denotes the importance coefficient (weight) of the $j$-th criterion, such that $\sum_{j=1}^{n} k_{j}=1$;

- $s$ denotes the concordance threshold; and

- $v_{j}$ denotes the veto threshold of the $j$-th criterion.

The conditions for stating that $a_{x}$ outranks $a_{y}$ (denoted $a_{x} S a_{y}$ ) depend on the method:

ELECTRE I (Roy, 1968; Roy and Bouyssou, 1993):

$$
a_{x} S a_{y} \Leftrightarrow c\left(a_{x}, a_{y}\right)=\sum_{j: \Delta_{j} \geq 0} k_{j} \geq s \wedge \forall j, \Delta_{j} \geq-v_{j} .
$$

ELECTRE IS (Roy and Skalka, 1984; Roy and Bouyssou, 1993):

Besides the previous parameters, let $q_{j}$ and $p_{j}$ denote the indifference and preference thresholds, respectively, of the $j$-th criterion. Then,

$$
\begin{aligned}
& a_{x} S a_{y} \Leftrightarrow \\
& \quad c\left(a_{x}, a_{y}\right)=\sum_{j} k_{j} c_{j}\left(a_{x}, a_{y}\right) \geq s \wedge \forall j, \Delta_{j} \geq-v_{j}+q_{j} w_{j}\left(a_{x}, a_{y}\right),
\end{aligned}
$$

where

$$
\begin{aligned}
& c_{j}\left(a_{x}, a_{y}\right)= \begin{cases}0, & \text { if } \Delta_{j}<-p_{j} ; \\
\left(p_{j}+\Delta_{j}\right) /\left(p_{j}-q_{j}\right), & \text { if }-p_{j} \leq \Delta_{j}<-q_{j} ; \\
1, & \text { if } \Delta_{j} \geq-q_{j} ;\end{cases} \\
& \text { and } w_{j}\left(a_{x}, a_{y}\right)=\frac{1-c\left(a_{x}, a_{y}\right)-k_{j}}{1-s-k_{j}} \\
& \text { or } w_{j}\left(a_{x}, a_{y}\right)=\frac{1-c\left(a_{x}, a_{y}\right)}{1-s} \text { (original version). }
\end{aligned}
$$


A third option is to use the credibility index as defined for the ELECTRE III method:

$$
a_{x} S a_{y} \Leftrightarrow \sigma\left(a_{x}, a_{y}\right) \geq s \text { (see Roy and Bouyssou, 1993, for details). }
$$

Note that we may generalize and write

$$
\begin{aligned}
& r\left(a_{x}, a_{y}\right)=\min \left\{c\left(a_{x}, a_{y}\right)-s, \Delta_{j}+v_{j}(j=1, \ldots, n)\right\}(\text { ELECTRE I), } \\
& r\left(a_{x}, a_{y}\right)=\min \left\{c\left(a_{x}, a_{y}\right)-s, \Delta_{j}+v_{j}-q_{j} w_{j}\left(a_{x}, a_{y}\right)(j=1, \ldots, n)\right\} \\
& \quad(\text { ELECTRE IS }), \text { or } \\
& r\left(a_{x}, a_{y}\right)=\sigma\left(a_{x}, a_{y}\right)-s \text { (ELECTRE based on credibility indices). }
\end{aligned}
$$$$
a_{x} S a_{y} \Leftrightarrow r\left(a_{x}, a_{y}\right) \geq 0,
$$

The following relations can be obtained from the outranking relation:

$a_{x} P a_{y}\left(a_{x}\right.$ is preferred to $\left.a_{y}\right) \Longleftrightarrow a_{x} S a_{y} \wedge \neg\left(a_{y} S a_{x}\right)$;

$a_{x} I a_{y}$ ( $a_{x}$ is indifferent to $\left.a_{y}\right) \Longleftrightarrow a_{x} S a_{y} \wedge a_{y} S a_{x}$;

$a_{x} R a_{y}\left(a_{x}\right.$ is incomparable to $\left.a_{y}\right) \Longleftrightarrow \neg\left(a_{x} S a_{y}\right) \wedge \neg\left(a_{y} S a_{x}\right)$.

The exploitation of the outranking relation finds a subset of actions, as small as possible, yet containing the most preferred one. This "kernel" $K \subseteq A$ is such that actions outside of $K$ are outranked by at least one action in $K$ (hence justifying its exclusion) and no action in $K$ is outranked by another action in $K$ that could exclude the former. If the graph representing the outranking relation contains cycles, it is necessary to regard each cycle as a single action (or a class of indifferent actions) to guarantee the existence of a kernel $K$ satisfying those two conditions (for details see Roy and Bouyssou, 1993).

\section{ROBUST CONCLUSIONS CONCERNING THE OUTRANKING RELATION}

Roy (1998) presented a framework defining the concept of robust conclusion as a formalized premise that is true for all the acceptable combinations of parameter values. He also introduced the notions of approximately robust conclusion (if it is true "almost everywhere") and pseudo-robust solution (if not perfectly formalized). In a different framework, Vincke (1999) defines the concepts of robust solutions and robust methods. For Vincke, a robust solution is one that is "close" (in some formalized manner) to all the solutions that correspond to admissible combinations of parameter values.

Robustness analysis contrasts with sensitivity analysis, conducted after obtaining a result, which determines how much may each parameter vary (often changing only one at a time) without leading to a different result. Although useful, these sensitivity analyses require an initial value for each parameter and focus on the result found initially, hence ignor- 
ing other interesting conclusions that might have been found with other initial values. This is different from global sensitivity analysis, which ascertains how may the variability in a model's output be partitioned into model input's variability (see Saltelli et al., 1999).

Let $T$ denote the set of all acceptable combinations of values for the parameters (performances, importance coefficients, veto thresholds,...). We will use Roy's definition of a robust conclusion, although there is a further distinction (introduced in Dias and Clímaco, 1999) that we deem useful when using decision aid methods:

- An absolute robust conclusion is a premise intrinsic to one of the actions, which is valid for every combination in $T$ (e.g. "the value of action $a_{x}$ is greater than 0.7 " in an additive aggregation model).

- A (relative) binary robust conclusion is a premise concerning a pair of actions, which is valid for every combination in $T$ (e.g. " $a_{x}$ outranks $a_{y}$ " in ELECTRE).

- A (relative) unary robust conclusion is a premise concerning one action but relative to others, which is valid for every combination in $T$ (e.g. " $a_{x}$ belongs to the kernel" in ELECTRE).

We will illustrate the methodology we advocate through the following fictitious example:

Example 1. Four actions are being compared according to five criteria (to be maximized) using ELECTRE I. Table 1.1 displays their performances, as well as the veto thresholds chosen for the criteria.

\begin{tabular}{l|rrrrr|}
\multicolumn{1}{c}{} & \multicolumn{1}{c}{$g_{1}$} & $g_{2}$ & $g_{3}$ & $g_{4}$ & $g_{5}$ \\
\cline { 2 - 6 }$a_{1}$ & 40 & 20 & 20 & 30 & 30 \\
$a_{2}$ & 30 & 30 & 30 & 20 & 10 \\
$a_{3}$ & 20 & 40 & 20 & 40 & 20 \\
$a_{4}$ & 20 & 10 & 40 & 10 & 40 \\
\cline { 2 - 6 }$v_{j}$ & 40 & 25 & 15 & 40 & 40 \\
\cline { 2 - 6 } & & & & &
\end{tabular}

Table 1.1 Performances and veto thresholds for Example 1

Consider that the importance of the last two criteria has been fixed $\left(k_{4}=0.1\right.$ and $\left.k_{5}=0.2\right)$, hence there is imprecise information concerning only the remaining three criteria. Suppose that this information leads to the following constraints defining $T$ :

$$
k_{1} \in[0.1,0.4], k_{2} \in[0.1,0.4], k_{3} \in[0.1,0.2], k_{1}+k_{2}+k_{3}=0.7 .
$$

Roy has suggested testing the robustness of a conclusion in a finite number of sample combinations $T_{s}$ from $T$ (Roy and Bouyssou, 1993; Roy, 1998): a few values are chosen for each parameter (e.g. maxi- 


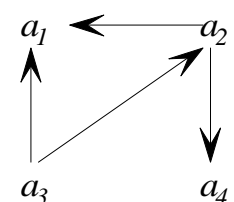

$k=(.1, .4, .2, .1, .2)$ $N=\left\{a_{3}, a_{4}\right\}$

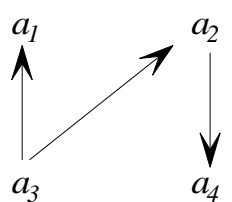

$k=(.2, .4, .1, .1, .2)$

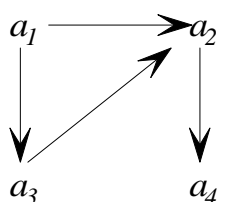

$k=(.275, .275, .15, .1, .2)$ $N=\left\{a_{1}, a_{4}\right\}$

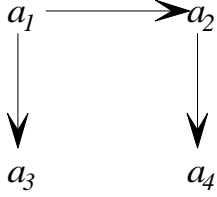

$k=(.4, .2, .1, .1, .2)$

$N=\left\{a_{1}, a_{4}\right\}$

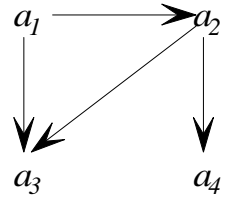

$k=(.4, .1, .2, .1, .2)$

$N=\left\{a_{1}, a_{4}\right\}$

Figure 1.1 Outranking relations (arrows denote outranking) and kernels for the combinations in $T_{s}$

mum, central and minimum); then, the sample consists of the admissible combinations of such parameter values. However, when the constraints defining $T$ inter-relate the parameter values, the set of admissible combinations taken as a sample may not be representative enough. An alternative sample could consist of the extreme points of $T$ (assuming it is a polytope), together with one or more points in its interior, e.g. $T_{s}=\{(.1, .4, .2),(.4, .1, .2),(.2, .4, .1),(.4, .2, .1),(.275, .275, .15)\}$. If we choose the latter sample $T_{s}$ and compute ELECTRE I's results for each point we will find the outranking relations and kernels in Figure 1.1 for a concordance level $s=0.55$.

Now, considering the final results of the method (i.e. the kernel $K$ ), there are only two (unary robust) conclusions that hold for all the combinations in $T_{s}$ : " $a_{4} \in K$ " and " $a_{2} \notin K$ ". However, if we look for binary robust conclusions concerning the outranking relation, we will find that $a_{2}$ always outranks $a_{4}$, whereas $a_{4}$ never outranks $a_{2}$ ! This paradox, resulting from the intransitivity of the outranking relation and the rules for forming the kernel, forced us to ponder which kind of robust conclusion should the analysis focus on. This example should not be dismissed on the grounds that it deals with a discrete set of combinations. Indeed, a discrete $T$ may correspond to a set of future scenarios or to a 
group of DMs that decides to form $T$ as the union of the combinations of values that each of them deems worth considering. (NOTE: if we considered $T$ instead of $T_{s}$, " $a_{4} \in K$ " and " $a_{2} \notin K$ " would hold for every combination exept $(.15, .35, .2)$, which lays on the boundary of $T$, whereas " $a_{2} S a_{4}$ " and "not $a_{4} S a_{2}$ " would always hold).

To our opinion, searching for binary robust conclusions concerning the outranking relation fits well into the ELECTRE's philosophy of confronting actions in pairs. Moreover, this has the advantage of being more transparent to the DMs to the extent that the concept of outranking is easier to understand than the concept of kernel. It also avoids the embarrassment of electing a winner ( $a_{4}$ in the previous example) in a situation where there is another action $\left(a_{2}\right)$ that is preferable to it (outranks it without being outranked) according to all the combinations of values for the parameters.

Given an ordered pair of actions $\left(a_{x}, a_{y}\right)$, from (1), considering $r\left(a_{x}, a_{y}\right)$ as a function of a combination of parameter values, we may test the robustness of conclusions concerning the outranking relation as follows:

- " $a_{x} S a_{y}$ " is robust (denoted $\left.a_{x} S^{R} a_{y}\right) \Leftrightarrow$

$$
\min \left\{r\left(a_{x}, a_{y}, t\right): t \in T\right\} \geq 0 ;
$$

- "not $a_{x} S a_{y}$ " is robust (denoted $\left.a_{x} N^{R} a_{y}\right) \Leftrightarrow$

$$
\max \left\{r\left(a_{x}, a_{y}, t\right): t \in T\right\}<0 .
$$

Therefore, we can uncover robust conclusions by maximizing and minimizing $r\left(a_{x}, a_{y}, t\right)$ (i.e. finding its range) in the domain $T$, for the multiple ordered pairs of actions (note that the range of $r\left(a_{x}, a_{y}, t\right)$ bears no information concerning the range of $r\left(a_{y}, a_{x}, t\right)$ ).

Maximizing and minimizing $r($.$) is often straightforward, even if this$ function corresponds to credibility indices, provided that the criteria weights can be varied independently of the thresholds and all constraints are linear. This is a reasonable assumption given the different nature of the parameters, but if it is violated the optimization does not become impossible: it becomes only harder to perform. The difficulty of the problems to be solved ranges from linear programming (ELECTRE I and original version of ELECTRE IS) to the optimization of non-linear quasiconcave functions (more recent version of ELECTRE IS and credibility indices). For details concerning the latter see Dias and Clímaco (1999).

Back to Example 1, since only the importance coefficients are variable, we would maximize and minimize the concordance indices $c($.$) , subject$ to (2). The range for the concordance indices concerning the pairs where the outranking is not vetoed is presented in Table 1.2. 


\begin{tabular}{l|cccc|}
\multicolumn{1}{c}{} & \multicolumn{1}{c}{$a_{1}$} & \multicolumn{1}{c}{$a_{2}$} & \multicolumn{1}{c}{$a_{3}$} & $a_{4}$ \\
\cline { 2 - 5 }$a_{1}$ & - & {$[0.4,0.7]$} & {$[0.5,0.8]$} & veto \\
$a_{2}$ & {$[0.3,0.6]$} & - & {$[0.3,0.6]$} & {$[0.6,0.7]$} \\
$a_{3}$ & {$[0.4,0.7]$} & {$[0.4,0.7]$} & - & veto \\
$a_{4}$ & {$[0.3,0.4]$} & {$[0.3,0.4]$} & veto & - \\
\cline { 2 - 5 } & & & &
\end{tabular}

Table 1.2 Concordance ranges for Example 1

Thus, the following conclusions concerning $S$ are robust, in that they hold for every combination of weights satisfying the constraints, given the fixed veto thresholds and $s=0.55$ :

- $a_{1}$ and $a_{3}$ do not outrank $a_{4}$;

- $a_{2}$ outranks $a_{4}$;

- $a_{4}$ does not outrank any other action.

The binary relations $S^{R}$ and $N^{R}$, corresponding to the robust conclusions, define an interval outranking relation, bounded from below by $S^{R}$ and bounded from above by $\overline{N^{R}}$ (the complement of $N^{R}$ ), such that, if we let $S^{t}$ denote the outranking relation corresponding a combination $t$ of parameter values, $\forall t \in T, S^{R} \subseteq S^{t} \subseteq \overline{N^{R}}$.

\section{ENRICHING THE ROBUST CONCLUSIONS}

This section addresses the problem (that may arise frequently) of dealing with relations $S^{R}$ and $N^{R}$ that are too poor (i.e. they hold for few ordered pairs). The ideal way of enriching these relations (and thereby increasing the number of robust conclusions) is to reduce the imprecision concerning the inputs by asking the DMs for more information. This elicitation should proceed in an interactive manner, so that the results of the analysis at a given iteration may prompt the discussion concerning the following one. For instance, DMs may deem that $S^{R}$ (or $N^{R}$ ) should apply to a given pair $\left(a_{x}, a_{y}\right)$, based on their capacity to judge the actions holistically. They may also wish to know the combination of parameter values leading to a given minimum or maximum $r($.$) . Then,$ if they consider the values unacceptable, they could insert a constraint to make that combination become unfeasible.

Richer conclusions may also be obtained if the conditions (3) and (4) are somehow relaxed. Of course, in this case the conclusions will be more fragile and the argument for accepting them becomes less compelling. Next, we next propose two different types of relaxation. 
Type 1: Ignoring a small fraction of the volume of $T$

The DMs may regard as robust a conclusion that holds for almost all of the combinations in $T$. Assuming that $T$ is a compact set with nonempty interior we may formalize this affirmation as stating that DMs could accept as robust a conclusion that holds for a large proportion of the volume of $T$. Such a conclusion might be called "approximately robust", according to the definition of Roy (1998).

Let $\operatorname{Vol}\left(t \in T: r\left(a_{x}, a_{y}, t\right) \geq 0\right)$ denote the volume of $T$ where $a_{x} S a_{y}$, whereas $\operatorname{Vol}(T)$ denotes the volume of $T$. Let $\epsilon$ denote a tolerance as regards the relative volumes where each conclusion holds, such that $0<\epsilon<0.5$. Then, we may define the following relations to generalize (3) and (4) (When $T$ is a discrete set, these relations can be defined by counting the proportion of the elements in $T$ that yield $a_{x} S a_{y}$ ):

- $a_{x} S^{V}(\epsilon) a_{y} \Leftrightarrow \operatorname{Vol}\left(t \in T: r\left(a_{x}, a_{y}, t\right) \geq 0\right) / \operatorname{Vol}(T) \geq 1-\epsilon$

- $a_{x} N^{V}(\epsilon) a_{y} \Leftrightarrow \operatorname{Vol}\left(t \in T: r\left(a_{x}, a_{y}, t\right) \geq 0\right) / \operatorname{Vol}(T) \leq \epsilon$.

These relations are more general and flexible than $S^{R}$ and $N^{R}$ : they coincide with $S^{R}$ and $N^{R}$ when $\epsilon=0$ and become richer (i.e. hold for a larger number of pairs of actions) as $\epsilon$ increases.

Table 1.3 presents the relative volumes of $T$ where each outranking occurs concerning Example 1. If the DMs regard as robust any conclusion that holds for $95 \%$ of the acceptable combinations (setting $\epsilon=0.05$ ), then the following conclusions would be added to the list presented in the previous section:

- $a_{2}$ does not outrank $a_{1}$

- $a_{2}$ does not outrank $a_{3}$.

The conclusion " $a_{1}$ outranks $a_{3}$ " would also be accepted if the DMs increased $\epsilon$ up to 0.2 . Of course, DMs should be given an idea of the combinations in the region they are neglecting, which might contain the values that they consider the most adequate.

\begin{tabular}{l|cccc|}
\multicolumn{1}{c}{} & \multicolumn{1}{c}{$a_{1}$} & $a_{2}$ & $a_{3}$ & $a_{4}$ \\
\cline { 2 - 5 }$a_{1}$ & - & $60 \%$ & $80 \%$ & veto \\
$a_{2}$ & $5 \%$ & - & $5 \%$ & $100 \%$ \\
$a_{3}$ & $40 \%$ & $60 \%$ & - & veto \\
$a_{4}$ & $0 \%$ & $0 \%$ & veto & - \\
\cline { 2 - 5 } & & & &
\end{tabular}

Table 1.3 Percentage of combinations for which outranking occurs (Example 1) 
The volume of $T$ may be analytically computed in the case of a polyhedral $T$ (e.g. Lasserre, 1983; Büeler et al., 1998). An alternative approach is to compute approximate volumes using Monte-Carlo simulation (e.g. Charnetski and Soland, 1978).

A different idea to relax the conditions for robustness which has a similar rationale consists in "contracting" the set $T$ by replacing each linear constraint of the form

$\alpha_{i 1} t_{1}+\ldots+\alpha_{i k} t_{k} \geq \beta_{i}\left(t_{1}, \ldots, t_{k}\right.$ denotes the parameters; $i$ indexes the constraint)

by a constraint

$\alpha_{i 1} t_{1}+\ldots+\alpha_{i k} t_{k} \geq \beta_{i}+\epsilon$ ( $\epsilon$ represents the positive tolerance value).

This places more emphasis on the more central combinations of $T$, which is particularly indicated when the DMs reason in terms of strict inequalities (e.g. $k_{1}>k_{2}$, which the relaxation converts into $k_{1} \geq k_{2}+\epsilon$ ), but requires the DMs to code all the constraints in a similar manner so that they may attribute a meaning to the value of $\epsilon$.

Type 2: Introducing a tolerance when comparing $\mathbf{r}($.$) with 0$

The simplest relaxation of all is to consider a small non-negative tolerance $\epsilon$ when comparing $r($.$) with zero. This amounts to generalize (3)$ and (4) by writing

- $a_{x} S^{Z}(\epsilon) a_{y} \Leftrightarrow \min \left\{r\left(a_{x}, a_{y}, t\right): t \in T\right\} \geq-\epsilon$;

- $a_{x} N^{Z}(\epsilon) a_{y} \Leftrightarrow \max \left\{r\left(a_{x}, a_{y}, t\right): t \in T\right\}<\epsilon$.

This type of relaxation can be readily applied whether $T$ is a discrete set or not. The relations $S^{Z}(\epsilon)$ and $N^{Z}(\epsilon)$ coincide with $S^{R}$ and $N^{R}$ when $\epsilon=0$ and become richer as $\epsilon$ increases. However, there is an important difference to Type 1: the relations $S^{Z}(\epsilon)$ and $N^{Z}(\epsilon)$ are not guaranteed to be mutually exclusive. In fact, there will appear pairs of actions $\left(a_{x}, a_{y}\right)$ such that $a_{x} S^{Z}(\epsilon) a_{y}$ and $a_{x} N^{Z}(\epsilon) a_{y}$, as soon as $\epsilon$ exceeds the threshold

$$
\epsilon^{K}=\min _{\left(a_{x}, a_{y}\right) \in A^{2}}\left\{\max \left\{-\min _{t \in T} r\left(a_{x}, a_{y}, t\right), \max _{t \in T} r\left(a_{x}, a_{y}, t\right)\right\}\right\} .
$$

Therefore, this type of relaxation fits naturally into the four-valued logic framework of Tsoukiàs and Vincke (1997). Considering an ordered pair of actions $\left(a_{x}, a_{y}\right)$, the statement that $a_{x}$ outranks $a_{y}$, may be:

- "true", if $a_{x} S^{Z}(\epsilon) a_{y} \wedge \neg\left(a_{x} N^{Z}(\epsilon) a_{y}\right)$;

- "false", if $\neg\left(a_{x} S^{Z}(\epsilon) a_{y}\right) \wedge a_{x} N^{Z}(\epsilon) a_{y}$;

- "unknown", if $\neg\left(a_{x} S^{Z}(\epsilon) a_{y}\right) \wedge \neg\left(a_{x} N^{Z}(\epsilon) a_{y}\right)$;

- "contradictory", if $a_{x} S^{Z}(\epsilon) a_{y} \wedge a_{x} N^{Z}(\epsilon) a_{y}$. 
Concerning Example 1, if we consider $\epsilon=0.055$ (which is $10 \%$ of $s$ and lower than $\epsilon^{K}=0.15$ ), we would accept an outranking if the minimum concordance was not lower than $s-\epsilon=0.495$ and we would reject that outranking if the maximum credibility was lower than $s+\epsilon=0.605$ (or if veto occurred). Hence, we would reach the same conclusions as when we used a Type 1 relaxation with $\epsilon=0.2$.

We believe that both types of relaxation are adequate and provide a compelling rationale (if $\epsilon$ is small) for accepting or rejecting an outranking. The second type of relaxation may even be combined with the first type. This second type of relaxation is very easy to perform (after the ranges $r($.$) have been computed) and places the emphasis on the output$ rather than the inputs. It also allows contradiction, which enables a richer analysis.

It is important to note that these relaxations are intended to be used in an interactive manner, where the DMs may experiment with the different types and with different values for $\epsilon$, with the objective of acquiring insight and of being able to provide new information. Let us also note that we have used the function $r($.$) to allow a more general presentation$ of our approach. A possible drawback is that $r($.$) is the minimum be-$ tween different aspects concerning concordance and discordance (veto) when using ELECTREs I or IS, which can make the value of $\epsilon$ somewhat difficult to interpret in the definitions of $S^{Z}(\epsilon)$ and $N^{Z}(\epsilon)$ (relaxation of Type 2). However, this is not important, because:

- $S^{R}, N^{R}$ and their relaxations can be redefined to deal with concordance and discordance separately;

- the performances can be normalized to be comparable, as in the original version of ELECTRE I;

- the function $r($.$) may be defined to deal with discordance and/or$ concordance in terms of relative deviation, e.g.

$$
r\left(a_{x}, a_{y}\right)=\min \left\{c\left(a_{x}, a_{y}\right)-s,\left(\Delta_{j}+v_{j}\right) / v_{j}(j=1, \ldots, n)\right\} .
$$

\section{EXPLOITING THE ROBUST CONCLUSIONS}

The most important goal of decision aid is perhaps the insight it generates. It may even happen that the best action becomes obvious once the DMs have learned enough about the situation and their preferences. In this perspective, finding robust conclusions (possibly relaxed ones) 
concerning the outranking relation $S$ yields the most important benefit. However, the DMs often need a structured approach (exploitation procedure) to select an action.

The exploitation of the robust conclusions in the context of a choice problem may be conducted by various means. A very important aspect is that this exploitation should not be isolated from the construction of the outranking relation. Instead, the exploitation of the robust conclusions and the identification of the results exhibiting higher variability (i.e. the pairs of actions for which the range of $r($.$) is wider) should prompt$ the DMs to revise the information that defines $T$, possibly reducing the amount of imprecision, which in turn leads to a new set of robust conclusions and a new iteration of the exploitation process, and so forth.

The literature on outranking relations offers some ideas to address our exploitation problem, as the following list demonstrates:

\section{Exploiting the relations $S$ and $N$}

Let us first consider that a Type 2 relaxation has been chosen, meaning that the robust conclusions allow to consider outrankings as "true", "false", "unknown" or "contradictory". Greco et al. (1997) propose the use of a score-based net flow procedure. Each action $a_{x} \in A$ would get the score

$$
\begin{aligned}
& s^{n f}\left(a_{x}\right)= \\
& \quad \#\left\{a_{y} \in A: \neg\left(a_{x} N^{Z}(\epsilon) a_{y}\right)\right\}-\#\left\{a_{y} \in A: \neg\left(a_{y} N^{Z}(\epsilon) a_{x}\right)\right\}+ \\
& \quad+\#\left\{a_{y} \in A: \neg\left(a_{y} S^{Z}(\epsilon) a_{x}\right)\right\}-\#\left\{a_{y} \in A: \neg\left(a_{x} S^{Z}(\epsilon) a_{y}\right)\right\} .
\end{aligned}
$$

Tsoukiàs and Vincke (1997) suggest that the "true" and "not false" relations could be separately exploited by some procedure to produce two rankings, which would be combined afterwards.

Considering now that a Type 1 relaxation is being used, or even no relaxation at all, the robust conclusions allow to consider the outranking of a given action over some other as "true" (if $S$ is robust), "false" (if $N$ is robust), or "unknown" (remaining cases). In this case, we are in presence of an interval outranking relation bounded by $S^{R}$ (or its relaxation) and $\overline{N^{R}}$ (or the complement of the relaxation of $N^{R}$ ), with $S^{R} \subseteq \overline{N^{R}}$. This means that the exploitation procedure of ELECTRE II (e.g. see Roy and Bouyssou, 1993: 409-415) can be used, considering that $S^{R}$ is the strong outranking relation, whereas $\overline{N^{R}}$ is the weak one. The two procedures outlined above can also be used, since they do not differentiate "unknown" from "contradictory" outrankings.

Notice that although we are interested in choice problems, all these procedures provide a ranking of the actions. 


\section{Exploiting valued outranking relations}

It is straightforward to use a relaxation of Type 1 to define a valued (fuzzy) outranking relation. Given an ordered pair $\left(a_{x}, a_{y}\right)$ of actions, the credibility of the statement " $a_{x}$ outranks $a_{y}$ " is equal to the proportion of $T$ 's volume where such outranking occurs (this idea of associating volumes with a valued relation is also present in Bana e Costa and Vincke, 1995). The credibility is maximum when $a_{x} S^{R} a_{y}$, it is minimum when $a_{x} N^{R} a_{y}$, and it has an intermediate value for the remaining cases. There are many methods to exploit binary valued relations, namely the net-flow procedure (Bouyssou, 1992), the min procedure (Pirlot, 1995), and ELECTRE III's distillation algorithms (Roy and Bouyssou, 1993).

\section{Exploiting a single outranking relation}

A different idea is to work with a single outranking relation $S^{M}$ and then exploit it to find a kernel according to the ELECTRE I/IS methods. This outranking relation could be $S^{R}$ or $\overline{N^{R}}$, if one of these relations is rich enough to exploit. Otherwise, DMs could consider the relaxation of $S^{R}$ or the complement of the relaxation of $N^{R}$. If a relaxation of Type 1 is used then $S^{R} \subseteq S^{M} \subseteq \overline{N^{R}}$. However, note that the relation $S^{M}$ might not correspond to a combination of parameter values in $T$.

Another possibility is to consider a "central" combination $t^{c} \in T$ and to exploit the outranking relation $S^{M}$ that this combination yields $\left(S^{R} \subseteq S^{M} \subseteq \overline{N^{R}}\right)$. The central combination $t^{c} \in T$ may be computed by following one of two approaches: $t^{c}$ may be chosen as a combination (there may be several) maximizing the minimum slack among the inequality constraints defining $T$; or $t^{c}$ may be chosen as the centroid of $T$ (Solymosi and Dombi, 1986), which is very easily computed when $T$ is defined by a ranking of the values for the parameters.

The main objective of using these exploitation techniques should be to prompt the DMs to revise their inputs and provide more information. Hence, experimenting with several of these techniques could enable a richer analysis. The exploitation can also be used to put an end to the analysis, particularly when $S^{R}$ is close to $\overline{N^{R}}$.

\section{ILLUSTRATIVE EXAMPLE}

As an illustration, let us consider the choice of a machine to sort packages, a problem faced by the French postal service (presentation based on Roy and Bouyssou, 1993: 501-541). In that study, ELECTRE IS was used to compare 9 actions according to 12 criteria (Table 1.4).

The criteria weights used in the original example are depicted in the $k_{j}$ row of Table 1.4. These weights were chosen to satisfy the following system, which reflects the opinion of the DMs: 


\begin{tabular}{ccccccccccccc}
\hline & $g_{1}$ & $g_{2}$ & $g_{3}$ & $g_{4}$ & $g_{5}$ & $g_{6}$ & $g_{7}$ & $g_{8}$ & $g_{9}$ & $g_{10}$ & $g_{11}$ & $g_{12}$ \\
\hline$a_{1}$ & 75 & 69 & 68 & 70 & 82 & 72 & 86 & 74 & $-15,23$ & 83 & 76 & 29 \\
$a_{2}$ & 81 & 60 & 82 & 70 & 66 & 52 & 86 & 60 & $-15,7$ & 83 & 76 & 71 \\
$a_{3}$ & 77 & 60 & 82 & 50 & 66 & 60 & 86 & 60 & -15 & 83 & 82 & 71 \\
$a_{4}$ & 73 & 57 & 82 & 90 & 75 & 61 & 93 & 60 & $-15,55$ & 83 & 71 & 29 \\
$a_{5}$ & 76 & 46 & 55 & 90 & 48 & 46 & 93 & 60 & $-36,68$ & 83 & 50 & 14 \\
$a_{6}$ & 75 & 63 & 68 & 90 & 98 & 63 & 78 & 61 & $-22,9$ & 100 & 68 & 57 \\
$a_{7}$ & 73 & 63 & 68 & 70 & 98 & 86 & 78 & 61 & $-19,58$ & 100 & 74 & 57 \\
$a_{8}$ & 77 & 31 & 41 & 50 & 59 & 79 & 71 & 60 & $-15,47$ & 67 & 76 & 86 \\
$a_{9}$ & 96 & 69 & 41 & 70 & 49 & 60 & 57 & 60 & $-13,99$ & 83 & 50 & 86 \\
\hline$q_{j}$ & 5 & 5 & 5 & 5 & 5 & 8 & 10 & 0 & 1 & 10 & 5 & 10 \\
$v_{j}$ & 50 & 50 & 40 & 100 & 40 & 25 & 100 & 50 & 5 & 100 & 30 & 50 \\
$k_{j}$ & $\frac{3}{39}$ & $\frac{2}{39}$ & $\frac{5}{39}$ & $\frac{3}{39}$ & $\frac{3}{39}$ & $\frac{5}{39}$ & $\frac{2}{39}$ & $\frac{2}{39}$ & $\frac{5}{39}$ & $\frac{1}{39}$ & $\frac{5}{39}$ & $\frac{3}{39}$ \\
\hline
\end{tabular}

Table 1.4 Performances (to be maximized) and thresholds for the example by Roy and Bouyssou (the preference thresholds $p_{j}$ coincide with $q_{j}$ )

(i) $k_{10}<k_{2}=k_{7}=k_{8}<k_{1}=k_{4}=k_{5}<k_{3}=k_{6}=k_{9}=k_{11}$,

(ii) $k_{10} \leq k_{12} \leq k_{11}$,

(iii) $k_{1}=k_{2}+k_{10}$,

(iv) $k_{11}=k_{1}+k_{2}$,

(v) $k_{j} \geq 0(j=1, \ldots, 12)$.

The original study set $s=0.7$, although it admitted that $s \in[0.63,0.73]$ when performing a robustness analysis a posteriori. The outranking relation from the original study is depicted in Figure 1.2. Based on this relation, $a_{5}, a_{6}$, and $a_{8}$ can obviously be excluded, while $a_{1}$ justifies the exclusion of $a_{2}, a_{3}$, and $a_{4}$, which form an indifference class. Hence, the kernel is $K=\left\{a_{1}, a_{7}, a_{9}\right\}$.

In our example, we will proceed by considering the imprecise information defined by the constraints above and see what conclusions may be drawn. We will use the same values of the original study for the thresholds associated with the criteria (rows $q_{j}$ and $v_{j}$ in Table 1.4). The set $T$ is defined by the constraints (i)-(v), the constraint $\sum_{j=1}^{n} k_{j}=1$ (which is not restrictive), and the bounds $s \in[0.63,0.73]$.

Let us now define $r($.$) to account for discordance as a ratio to the veto$ thresholds, in order to attribute some meaning to inter-criteria comparisons of discordance:

$$
r\left(a_{x}, a_{y}\right)=\min \left\{c\left(a_{x}, a_{y}\right)-s, \frac{\Delta_{j}+v_{j}-q_{j} w_{j}\left(a_{x}, a_{y}\right)}{v_{j}}(j=1, \ldots, n)\right\} .
$$




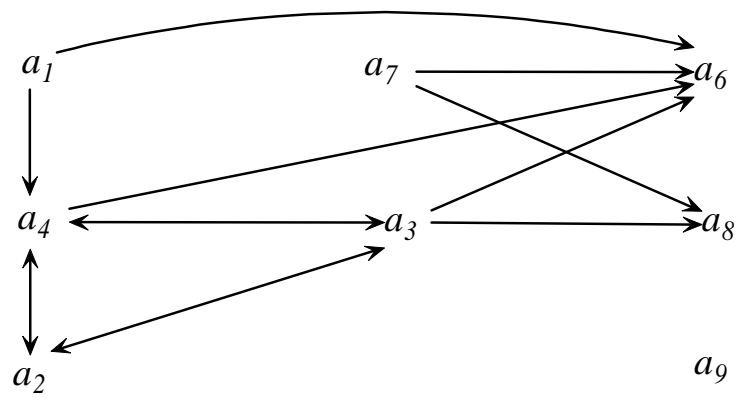

Figure 1.2 Outranking relation for the example by Roy and Bouyssou ( $a_{5}$ does not appear since it is outranked by every other action).

\begin{tabular}{lccccc|}
\multicolumn{1}{c}{$a_{1}$} & $a_{2}$ & $a_{3}$ & $a_{4}$ & $a_{5}$ \\
\cline { 2 - 6 }$a_{1}$ & - & {$[-.08, .04]$} & {$[-.12, .01]$} & {$[.05, .18]$} & {$[.18, .31]$} \\
$a_{2}$ & {$[-.28, .05]$} & - & {$[.13, .25]$} & {$[-.03, .12]$} & {$[.18, .31]$} \\
$a_{3}$ & {$[-.16, .01]$} & {$[.18, .31]$} & - & {$[.1, .25]$} & {$[.08, .31]$} \\
$a_{4}$ & {$[-.09, .09]$} & {$[.01, .12]$} & {$[-.03, .08]$} & - & {$[.27, .37]$} \\
$a_{5}$ & {$[-4.34,-3.82]$} & {$[-3.95,-3.57]$} & {$[-4.29,-3.82]$} & {$[-4.18,-3.71]$} & - \\
$a_{6}$ & {$[-1.21,-.8]$} & {$[-1.10,-.75]$} & {$[-1.14,-.83]$} & {$[-.81,-.59]$} & {$[.2, .33]$} \\
$a_{7}$ & {$[-.09, .08]$} & {$[-.24, .01]$} & {$[-.48,-.17]$} & {$[-.26,0]$} & {$[.13, .25]$} \\
$a_{8}$ & {$[-.23,-.07]$} & {$[-.31,-.15]$} & {$[-.38,-.19]$} & {$[-.31,-.15]$} & {$[-.09, .06]$} \\
$a_{9}$ & {$[-.67,-.15]$} & {$[-.31,-.14]$} & {$[-.44,-.23]$} & {$[-.38,-.19]$} & {$[-.02, .13]$} \\
\cline { 2 - 6 }
\end{tabular}

\begin{tabular}{lcccc|}
\multicolumn{1}{c}{} & $a_{6}$ & $a_{7}$ & $a_{8}$ & $a_{9}$ \\
\cline { 2 - 5 }$a_{1}$ & {$[-.05, .23]$} & {$[-.09, .16]$} & {$[-.23,-.14]$} & {$[-.38,-.26]$} \\
$a_{2}$ & {$[-.12, .06]$} & {$[-.69,-.45]$} & {$[-.3,-.03]$} & {$[-.11, .08]$} \\
$a_{3}$ & {$[.01, .15]$} & {$[-.60,-.23]$} & {$[.01, .23]$} & {$[-.17, .01]$} \\
$a_{4}$ & {$[-.05, .16]$} & {$[-.67,-.25]$} & {$[-.33,-.22]$} & {$[-.42,-.29]$} \\
$a_{5}$ & {$[-2.81,-2.32]$} & {$[-3.47,-2.98]$} & {$[-3.90,-3.56]$} & {$[-4.10,-3.79]$} \\
$a_{6}$ & - & {$[-.57,-.17]$} & {$[-1.05,-.74]$} & {$[-1.15,-.91]$} \\
$a_{7}$ & {$[.18, .31]$} & - & {$[.01, .18]$} & {$[-.48,-.24]$} \\
$a_{8}$ & {$[-.2,-.1]$} & {$[-.2,-.1]$} & - & {$[-.12, .06]$} \\
$a_{9}$ & {$[-.54,-.40]$} & {$[-1.20,-.56]$} & {$[-.42,-.03]$} & - \\
\cline { 2 - 5 }
\end{tabular}

Table 1.5 Ranges for $r($.

According to our approach, we have to find the maximum and minimum $r\left(a_{x}, a_{y}, t\right)$, subject to $t \in T$, for all ordered pairs $\left(a_{x}, a_{y}\right) \in A \times A$ 
(Table 1.5). There are many robust conclusions that may be drawn from these results. Particularly, we may note that $a_{5}$ never outranks any other action and is always outranked by $a_{1}$ to $a_{7}$. The action $a_{6}$ never outranks any other (except $a_{5}$ ) and is always outranked by $a_{3}$ and $a_{7}$. Obviously, $a_{5}$ and $a_{6}$ are not contenders for the best action and can be deleted.

Figure 1.3 represents the relation $S^{R}$ through thick arrows and the complement of $N^{R}$ as segmented arrows. Hence, a thick arrow may be read as "always outranks", a segmented arrow may be read as "may outrank", and the absence of an arrow indicates "never outranks". These relations could be exploited by any of the techniques from the previous section. As an example, the exploitation of the relation $S^{R}$ according to the rules of ELECTRE I/IS leads to the kernel $K=\left\{a_{1}, a_{7}, a_{9}\right\}$, which is equal to the original study's.

To continue this example, let us suppose that the DMs were invited to think about the doubtful outrankings and they would answer that they were expecting that $a_{1}$ would outrank $a_{7}$. At this point, they could learn that the combination yielding the minimum $r\left(a_{1}, a_{7}\right)$ was $k_{1}=k_{4}=k_{5}=$ $0.08, k_{2}=k_{7}=k_{8}=k_{10}=0.04, k_{3}=k_{6}=k_{9}=k_{11}=k_{12}=0.12$, and $s=0.73$. Analyzing this information, suppose that the DMs would state that $k_{2}$ should not be less than $k_{12}$, which corresponds to a new constraint on $T$ (in this case, if they would state that $a_{1} \mathrm{~S} a_{7}$, then it could also be coded as a linear constraint). The ranges for the $r($. functions would become smaller, as shown in Table 1.6.

By now it is clear that $a_{1} S a_{7}$, although the DMs did not require it directly. Let us also suppose that the DMs would accept a Type 2 relaxation with a tolerance $\epsilon=0.03$. Figure 1.4 depicts the conclusions corresponding to $S^{Z}(0.03)$ and $N^{Z}(0.03)$. The decision process could then continue, either asking the DMs for more information (e.g. is $a_{1}$

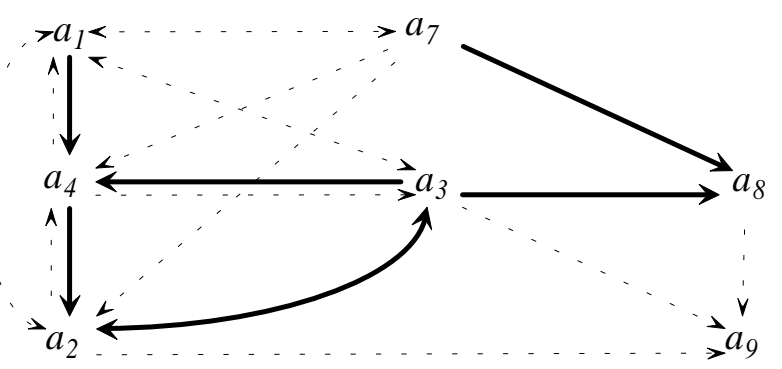

Figure 1.3 Relations "always outranks" and "may outrank". 


\begin{tabular}{l|ccccccc|}
\multicolumn{1}{c}{} & $a_{1}$ & $a_{2}$ & $a_{3}$ & $a_{4}$ & $a_{7}$ & $a_{8}$ & $a_{9}$ \\
\cline { 2 - 8 }$a_{1}$ & - & {$[-.04, .04]$} & {$[-.09, .01]$} & {$[.05, .17]$} & {$[.03, .16]$} & {$[-.19,-.14]$} & {$[-.34,-.26]$} \\
$a_{2}$ & {$[-.28, .02]$} & - & {$[.13, .24]$} & {$[-.03, .10]$} & {$[-.69,-.47]$} & {$[-.17,-.03]$} & {$[-.06, .08]$} \\
$a_{3}$ & {$[-.16,-.02]$} & {$[.18, .30]$} & - & {$[.1, .24]$} & {$[-.60,-.25]$} & {$[.07, .23]$} & {$[-.13, .01]$} \\
$a_{4}$ & {$[-.09, .07]$} & {$[.06, .12]$} & {$[.01, .08]$} & - & {$[-.58,-.25]$} & {$[-.29,-.22]$} & {$[-.39,-.29]$} \\
$a_{7}$ & {$[-.09, .06]$} & {$[-.18, .01]$} & {$[-.43,-.17]$} & {$[-.26,-.02]$} & - & {$[.07, .18]$} & {$[-.42,-.24]$} \\
$a_{8}$ & {$[-.23,-.10]$} & {$[-.31,-.17]$} & {$[-.38,-.20]$} & {$[-.31,-.17]$} & {$[-.20,-.11]$} & - & {$[-.12, .04]$} \\
$a_{9}$ & {$[-.67,-.20]$} & {$[-.31,-.16]$} & {$[-.44,-.25]$} & {$[-.38,-.20]$} & {$[-1.20,-.61]$} & {$[-.42,-.06]$} & - \\
\hline
\end{tabular}

Table 1.6 Ranges after additional constraint

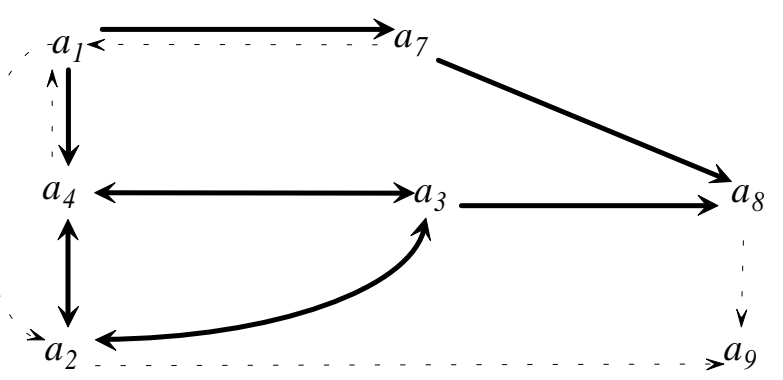

Figure 1.4 Relations "always outranks" and "may outrank" after additional constraint and accounting for a tolerance of 0.03 .

preferred to $a_{4}$ or indifferent to it?), or exploiting the relations obtained. Action $a_{1}$ would have the highest net-flow score and would appear at the top of ELECTRE II's exploitation ranking. It would also belong to the kernel if either $S^{Z}(0.03)$ or $N^{Z}(0.03)$ were exploited by the usual process in ELECTRE I/IS.

This example illustrates how it is possible to work with imprecise information as a means to obtain robust conclusions. In this case, the robust conclusions or slight relaxations of these conclusions are rich enough to advance towards their exploitation. The exploitation led to results very similar to those of the original study, but easier to justify, since we did not need to fix precise values for the parameters for which only imprecise information was available. However, the use of these results to elicit further information from the DMs (hence constraining $T$ ) would probably be yet more interesting. 


\section{CONCLUDING REMARKS}

Instead of bulldozing the difficulties and hesitation of the DMs, through a quest for the right combination of values for the parameters, we deem that imprecision should be accepted from the very beginning of the decision aid process. This allows to alleviate the DMs' cognitive burden at the beginning, postponing the most difficult questions to a stage when they are more familiar with the problem at hand and the decision aid method.

The analysis proposed here explores the consequences of the imprecise information that the DMs are able (or willing) to provide. We focus on exploration rather than aggregation, avoiding the computation of averages, median values, and other usual aggregation means. The exploration allows to discover which conclusions are robust and allows to identify which conclusions are more affected by the imprecision. This is particularly important in what regards the questions that can be posed to the DMs when more information is needed.

This paper does not propose a precise method. Rather, we feel that the array of tools to be used will depend on the problem at hand. A decision support system implementing several of these tools would hence be quite helpful. Most of the approaches proposed here have the possible drawback of demanding some computational effort (optimization or volume computation). However, the DMs will not perceive this for two reasons. On the one hand, today's low cost personal computers are sufficiently powerful to solve these problems in acceptable time for the problem dimensions usually found in practice. On the other hand, the DMs need only understand the results, and not the algorithmic details of their computation. The concept of a robust conclusion such as never outranks or always outranks is, of course, easy to apprehend. We might say that this is a case of using "hard" tools for a "softer" decision aid. 


\section{References}

Bana e Costa. C.A. and Ph. Vincke (1995). Measuring credibility of compensatory preference statements when trade-offs are interval determined, Theory and Decision 39, 127-155.

Bouyssou, D. (1992), Ranking methods based on valued preference relations: a characterization of the net-flow method, European Journal of Operational Research 60, 61-68.

Büeler, B., A. Enge and K. Fukuda (1998), Exact volume computation for polytopes: a practical study, in Polytopes - Combinatorics and Computation, DMV-Seminars, Birkhäuser Verlag (to appear).

Charnetski J.R. and R.M. Soland (1978). Multiple-attribute decision making with partial information: the comparative hypervolume criterion. Naval Research Logistics 25, 278-288.

Dias, L. C. and J. N. Clímaco (1999), On computing ELECTRE's credibility indices under partial information, Journal of Multi-Criteria Decision Analysis 8, 74-92.

Dias, L. C. and J. N. Clímaco (2000a), Additive aggregation with variable interdependent parameters: the VIP Analysis software, Journal of the Operational Research Society 51, 1070-1082.

Dias, L. C. and J. N. Clímaco (2000b), ELECTRE TRI for Groups with Imprecise Information on Parameter Values, Group Decision and Negotiation 9, 355-377.

Dias, L., V. Mousseau, J. Figueira and J. Clímaco (2000), An Aggregation/Disaggregattion approach to obtain robust conclusions with ELECTRE TRI, Cahier du LAMSADE, No. 174, Université ParisDauphine. (To appear in EJOR).

French, S. (1995), Uncertainty and imprecision: modelling and analysis, Journal of the Operational Research Society 46, 70-79. 
Greco, S., B. Matarazzo, R. Slowinski amd A. Tsoukiàs (1997), Exploitation of a rough approximation of the outranking relation, Cahier du LAMSADE, No. 152, Université Paris-Dauphine.

Lasserre, J.B. (1983), An analytical expression and an algorithm for the volume of a convex polyhedron in $\Re^{n}$, Journal of Optimization Theory and Applications 39, 363-377.

Miettinen, K. and P. Salminen (1999), Decision-aid for discrete multiple criteria decision making problems with imprecise data, European Journal of Operational Research 119, 50-60.

Mousseau, V. (1993), Problmes liés à l'evaluation de l'importance relative des critères en aide multicritère à la décision: refléxions théoriques, experimentation et implementation informatique, $\mathrm{PhD}$ Thesis, Université Paris-Dauphine.

Pirlot, M. (1995), A characterization of 'Min' as a procedure for exploiting valued preference relations and related results, Journal of MultiCriteria Decision Analysis 4, 37-56.

Roy, B. (1968), Classement et choix en présence de points de vue multiples (la méthode ELECTRE), Revue Informatique et Recherche Oprationelle, 2e. Anne, No. 8, 57-75.

Roy, B. (1989), Main sources of inaccurate determination, uncertainty and imprecision in decision models, Mathematical and Computer Modelling 12, 1245-1254.

Roy, B. (1991), The outranking approach and the foundations of ELECTRE methods, Theory and Decision 31, 49-73.

Roy, B. (1998), A missing link in OR-DA: robustness analysis, Foundations of Computing and Decision Sciences 23, 141-160.

Roy B. and Bouyssou, D. (1993), Aide multicritère à la décision: méthodes et cas, Economica, Paris.

Roy, B. e J. M. Skalka (1984), ELECTRE IS: aspects méthodologiques et guide d'utilisation, Document du LAMSADE, No. 30, Université Paris-Dauphine.

Saltelli, A., S. Tarantola and K. Chan (1999), A role for sensitivity analysis in presenting the results from MCDA studies to decision makers, Journal of Multi-Criteria Decision Analysis 8, 139-145.

Solymosi, T and J. Dombi (1986), A method for determining the weights of the criteria: the centralized weights, European Journal of Operational Research 26, 35-41.

Tsoukiàs, A. and Ph. Vincke (1997), Extended preference structures in multicriteria decision aid, in Clímaco, J. (ed.), Multicriteria analysis, Springer, 37-50.

Vincke, Ph. (1999), Robust solutions and methods in decision aid, Journal of Multi-Criteria Decision Analysis 8, 181-187. 